\title{
Transradial versus transfemoral intervention in ST-segment elevation myocardial infarction patients in Korean population
}

$\mathrm{Hu} \mathrm{Li} i^{1,2}$, Seung-Woon $\mathrm{Rha}^{2}$, Byoung Geol Choi ${ }^{2}$, Min Suk Shim², Se Yeon $\mathrm{Choi}^{2}$, Cheol Ung $\mathrm{Choi}^{2}$, Eung Ju Kim², Dong Joo $\mathrm{Oh}^{2}$, Byung Ryul Cho ${ }^{3}$, Moo Hyun Kim, Doo-Il Kim5, Myung-Ho Jeong ${ }^{6}$, Sang Yong $\mathrm{Yoo}^{7}$, Sang-Sik Jeong ${ }^{7}$, Byung Ok Kim ${ }^{8}$, Min Su Hyun ${ }^{9}$, Young-Jin Youn ${ }^{10}$, and Junghan Yoon ${ }^{10}$

\author{
${ }^{1}$ Department of Cardiovascular, \\ The Second Affiliated Hospital \\ of Kunming Medical University, \\ Kunming, China; ${ }^{2}$ Department \\ of Medicine, Graduate School, \\ Korea University College of \\ Medicine, Seoul; ${ }^{3}$ Department of \\ Cardiology, Kangwon National \\ University Hospital, Chuncheon; \\ ${ }^{4}$ Cardiovascular Center, Dong-A \\ University Hospital, Busan; \\ ${ }^{5}$ Cardiovascular Center, Inje \\ University Busan Paik Hospital, \\ Busan; ${ }^{6}$ Cardiovascular Center, \\ Chonnam National University \\ Hospital, Gwangju; ${ }^{7}$ Cardiovascular \\ Center, Gangneung Asan \\ Hospital, University of Ulsan \\ College of Medicine, Gangneung; \\ ${ }^{8}$ Cardiovascular Center, Inje \\ University Sanggye Paik Hospital, \\ Seoul; ' ${ }^{9}$ Cardiovascular Center, \\ Soonchunhyang University Seoul \\ Hospital, Seoul; ${ }^{10}$ Cardiovascular \\ Center, Wonju Severance Christian \\ Hospital, Yonsei University Wonju \\ College of Medicine, Wonju, Korea
}

Received: September 30, 2016 Revised : December 24, 2016 Accepted: January 9, 2017
Background/Aims: Transradial intervention (TRI) is becoming the preferred method over transfemoral intervention (TFI) because TRI is associated with lower incidence of major bleeding and vascular complications. However, there has been limited published data regarding the clinical outcomes of TRI versus TFI in Korean patients with ST-elevation myocardial infarction (STEMI).

Methods: A total of 689 consecutive STEMI patients who underwent primary percutaneous coronary intervention (PCI) with drug-eluting stents (DESs) from January to December of 2009 at nine university hospitals were enrolled in this study. Mid-term angiographic and 12-month cumulative clinical outcomes of the TRI group $(n=220,31.9 \%)$ were compared to those of the TFI group ( $n=469,28.1 \%)$.

Results: After propensity score matching, in-hospital complications and the 12-month major clinical outcomes during follow-up in the two groups were similar to each other. However, the incidence rates of repeat revascularization $(6.4 \%$ vs. $0.5 \%, p=0.003)$, target vessel revascularization $(6.4 \%$ vs. $0.5 \%, p=0.003)$, and major adverse cardiac events (MACE; $11.6 \%$ vs. $4.6 \%, p=0.018$ ) in the TFI group were higher than those in the TRI group during the 12-month of follow-up. Conclusions: In our study, TRI in STEMI patients undergoing primary PCI with DESs was associated with lower incidence of access site hematoma, 12-month repeat revascularization, and MACE compared to TFI. Therefore, TRI might play an important role in reducing bleeding complications while improving major clinical outcomes in STEMI patients undergoing primary PCI with DESs.

Keywords: Transradial intervention; Transfemoral intervention; ST elevation myocardial infarction; Vascular complications; Access site

\section{Correspondence to}

Seung-Woon Rha, M.D.

Cardiovascular Center, Korea University Guro Hospital, 148 Gurodong-ro, Guro-gu, Seoul 08308, Korea

Tel: $+82-2-2626-3020$

Fax: +82-2-864-3062

E-mail: swrha617@yahoo.co.kr 


\section{INTRODUCTION}

Transradial intervention (TRI) is known to be associated with fewer vascular complications and shorter duration of hospitalization than transfemoral intervention (TFI) $[1,2]$. In previous studies, TFI is associated with up to $10 \%$ of incidence rate of bleeding and vascular complications [3]. Primary percutaneous coronary intervention (PCI) for ST-elevation myocardial infarction (STEMI) is known to be associated with higher rates of bleeding complications, access site complications, and a need for transfusion in the TFI. These complications have been associated with poorer outcomes. They are also independent prognostic factors of mortality [4]. Other complications including arteriovenous fistula (AVF), pseudoaneurysm (PSA), acute arterial occlusion, and infection can also influence mortality of STEMI patients $[5,6]$.

Recently, TRI is becoming more widely used for routine PCI with contemporary drug-eluting stents (DESs) due to several advantages, including less bleeding, less vascular complications, lower mortality, and early ambulation. In Radial Versus Femoral Access for Coronary Angiography or Intervention (RIVAL) study, the TRI approach has shown a significantly lower 30-day mortality [7]. However, there has been limited published data regarding the clinical outcomes of TRI versus TFI in Korean patients with STEMI.

Therefore, the objective of this study was to compare in-hospital complications and major clinical outcomes of TRI with those of TFI in a series of Korean STEMI patients undergoing primary PCI and DESs with 12-month of follow-up.

\section{METHODS}

\section{TRI working group registry}

The Korean TRI working group (TRI-WG) registry was performed at nine university hospitals. These hospitals have high-volume patients and facilities for primary PCI and on-site cardiac surgery. Data were collected at each site by trained study coordinators with a standardized case report form. The study protocol was approved by the Institutional Review Board from each site, all patients provided written informed consent. Standardized definitions were used for all patient-related variables and clinical diagnoses. The study protocol was approved by the Ethics Committee at each participating institution.

\section{Study population}

A total of 4,785 patients who underwent PCI from January to December 2009 were retrospectively enrolled in the TRI-WG registry. A total of 936 eligible STEMI patients who underwent primary PCI with DESs were enrolled, patients were excluded if they had any of the following conditions: cardiogenic shock on admission, Killips grade IV cardiac function, hemodynamic supporting devices (intra-aortic balloon counterpulsation or extracorporeal membrane oxygenation), received bare metal stents, or received balloon angioplasty. A total of 689 patients were finally enrolled for this study and divided into two groups according to vascular access (transradial approach group, $\mathrm{n}=22 \mathrm{O}$; transfemoral approach group, $n=469$ ). To adjust baseline potential confounders, propensity score matched (PSM) analysis was performed using logistic regression model. After PSM, two well-balanced and well-matched groups (171 pairs, $n=342$, C-statistic $=0.792$ ) were generated and analyzed.

\section{$\mathrm{PCl}$ procedure and medical treatment}

Diagnostic angiography and PCI were performed through either the femoral or radial artery after administration of unfractionated heparin (70 to $100 \mathrm{U} / \mathrm{kg}$ ) to maintain activated clotting time of $>250$ seconds in patients during the procedure. Stents were deployed after balloon angioplasty. Cilostazol or platelet glycoprotein (GP) IIb/IIIa receptor blocker was used at the discretion of individual operator. A successful PCI was defined as the achievement of an angiographic residual stenosis of less than $30 \%$ in the presence of thrombolysis in myocardial infarction (TIMI) blood flow grade 3. During in-hospital period, patients received cardiovascular beneficial medications including $\beta$-blockers, renin angiotensin system inhibitors, calcium channel blockers, and statins. After discharge, patients were encouraged to stay on the same medications as they received in the hospital.

\section{Study definitions and clinical follow-up}

Cardiovascular risk factors and past history (age, sex, 
hypertension, dyslipidemia, smoking, diabetes mellitus, prior myocardial infarction [MI], chronic heart failure [HF], prior cerebrovascular disease, and peripheral arterial disease) were based on self-reports of patients. Assess site complications were defined as vascular complications that occurred in the access vessel such as hematomas (minor hematoma was defined as $<4 \mathrm{~cm}$ of hematoma induration and major hemato$\mathrm{ma} \geq 4 \mathrm{~cm}$ ), dissections, perforations, AVFs, and PSAs. Bleeding complications were defined as those previously described by Mehran et al. [8]. Procedure times were measured from the start of the puncture to the removal of the guiding catheters. Fluoroscopy times were measured automatically using fluoroscopes. Clinical endpoints were defined as those previously described by Cutlip et al. [9]. All deaths were considered as cardiac ones unless a non-cardiac cause was documented. Recurrent MI (Re-MI) was defined as recurrent symptoms of new ST-segment elevation or re-elevation of cardiac markers to a level of at least twice of the normal upper limit. Target lesion revascularization (TLR) was defined as an ischemia-induced PCI of the target lesion due to restenosis or re-occlusion within the stent or $5 \mathrm{~mm}$ in and adjacent of the distal or proximal segment. Target vessel revascularization (TVR) was defined as a clinically driven PCI of the target lesion or any segment of the coronary artery containing the target lesion. Total major adverse cardiac events (MACEs) was defined as the composite of total death, non-fatal Re-MI, and TVR. All participants were required to visit the outpatient Department of Cardiology at the end of the first month, every 3 to 6 months after the PCI procedure, and whenever angina-like symptoms occurred. Cumulative incidence of individual and composite clinical outcomes at 1-year of follow-up was compared between the two groups.

\section{Study end-points}

The primary study end-points were bleeding and vascular complications, procedural complications, and 30-day clinical outcomes after procedure. The secondary study end-points were cumulative incidence of individual and composite clinical outcomes during 1-year of clinical follow-up.

\section{Statistical analysis}

For continuous variables, differences between the two groups were evaluated by unpaired $t$ test or Mann-Whitney rank test. Data were expressed as mean \pm standard deviation of means. For discrete variables, differences were expressed as counts and percentages. They were analyzed with chi-square or Fisher exact test between the two groups when appropriate. To adjust for potential confounders, PSM analysis was performed using logistic regression model. We tested all available variables that could be of potential relevance, including age, male, cardiovascular risk factors (hypertension, diabetes, dyslipidemia, HF, chronic kidney disease, coronary artery disease, stroke, peripheral artery disease, history of MI or PCI, and current smoker), angiographic factors (target vessels [left main, left anterior descending, left circumflex, right coronary artery], lesion type [B2, C], chronic total occlusion [CTO], bifurcation, diffuse long lesion, and calcification), and medications (cilostazol, angiotensin II receptor blockers, angiotensin-converting enzyme inhibitors, calcium channel blockers, $\beta$-blockers, diuretics, and statin). Patients in the TRI group were then 1-to-1 matched to those in the TFI group according to propensity scores with the nearest available pair matching method. Subjects were matched with a caliper width of 0.05 . The procedure yielded 171 well-matched pairs. Cox-proportional hazard models were used to assess the hazard ratios in the two groups, the entire population, and the PSM population. The outcome of both groups were censored at a fixed point of 1 year ( 365 days) to avoid any bias caused by different follow-up duration. For all analyses, a two-sided $p$ value of less than 0.05 was considered as statistically significant. All statistical analyses were performed using SPSS version 20.0 (IBM Co., Armonk, NY, USA).

\section{RESULTS}

\section{Baseline clinical characteristics and laboratory findings}

A total of 4,785 patients were enrolled in the Korean TRIWG registry, including 936 patients who were diagnosed with STEMI. Among the 936 patients, 247 patient did not fulfill the inclusion criteria. Finally, 220 patient underwent PCI via TRI group while 469 patients underwent PCI via TFI group (Fig. 1). The baseline demographics, clinical characteristics, and laboratory findings of the two 


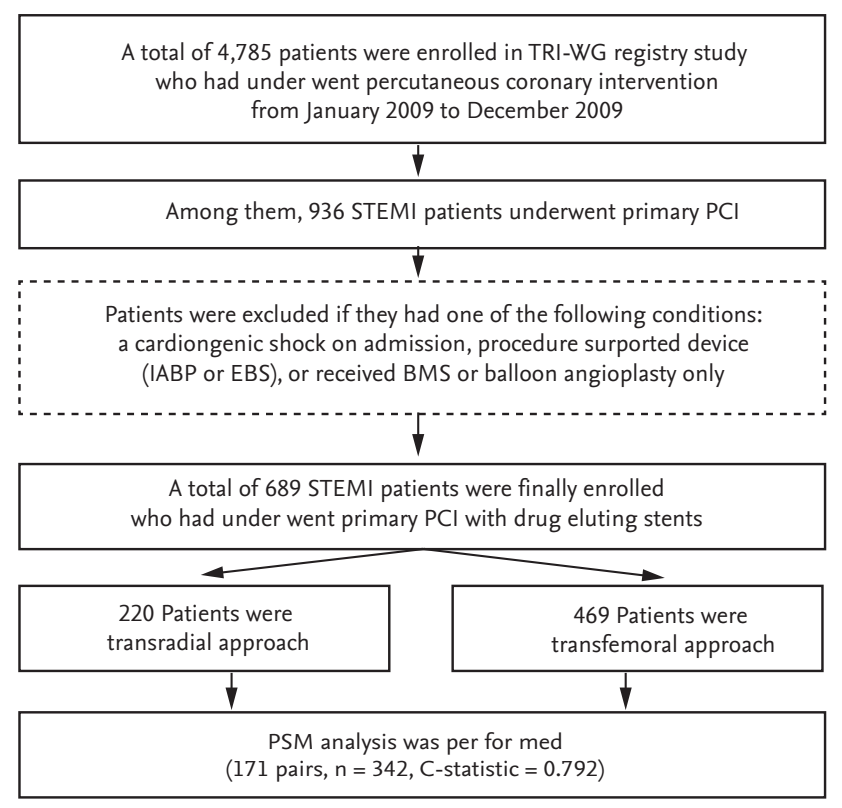

Figure 1. Study flow chart. TRI-WG, transradial intervention-working group; STEMI, ST-elevation myocardial infarction; PCI, percutaneous coronary intervention; IABP, intra-aortic balloon pump; EBS, emergency bypass system; BMS, bare metal stent; PSM, propensity score matching.

groups are summarized in Table 1. There were no significant differences in age, left ventricular ejection fraction, previous MI, or previous PCI. However, the prevalence rates of hypertension, diabetes, previous stroke, and current smoking were significantly higher than those in the TRI group. PSM yielded 171 pairs with well-balanced baseline characteristics. There was no significant differences in baseline characteristics between the TRI and TFI groups (Table 1).

\section{Baseline angiographic and procedural characteristics}

At baseline angiography, the TFI group had higher incidence rates of multivessel disease, left circumflex lesion, B2/C lesions, and CTO lesion than the TRI group. The TFI group used bigger sheath than the TRI group. In addition, more patients in the TRI group used closure devices than those in the TRI group. Image and physiologic study guided intervention and adjuvant balloon inflation were more frequent in the TRI group compared to those in the TFI group. Baseline angiographic and procedural characteristics after PSM were similar to each other between the two groups except that the incidence rates of smaller sheath use, image and physiology guided inter- vention, and adjuvant ballooning were higher in the TRI group. Contrast amount was higher in the TRI group than that in the TFI group. Procedure time and fluoroscopic time were also longer in the T'RI group (Table 2).

\section{Study primary end-points}

Major in-hospital and access site complications are listed in Table 3. The incidence rates of procedure-related acute and in-hospital complications in the two groups were similar to each other. The incidence of vascular access site hematoma in the TFI group was significantly higher than that in the TRI group. Although the incidence of major hematoma was similar to each other between the two groups, the incidence of minor hematomas was higher in the TFI group than that in the TRI group. No major life-threatening bleeding complications were observed in either group. Furthermore, the incidence of anemia requiring transfusion was similar to each other between the two groups. After PSM, the incidence of minor hematoma remained more frequent in the TFI group. However, all other procedural and in-hospital complications were similar to each other between the two groups (Table 3).

\section{Study secondary end-points}

In the entire patient population before PSM, the incidence rates of recurrent MI (particularly non-STEMI [NSTEMI]) and repeat revascularization including TLR and TVR were higher in the TFI group than those in the TRI group during the 12-month of follow-up. After PSM, the incident rates of major clinical outcomes including mortality, recurrent MI, TLR, and stent thrombosis rates during the 12-month of follow-up showed no disparity between the two groups. However, the incidence of TVR and MACE were higher in the TFI group (Table 4).

Results of multivariate logistic regression analysis of individual and composite major clinical outcomes during the 12-month of follow-up are shown in Table 5. The incident rates of TVR and MACE were higher in the TFI group after adjustment. In PSM patients, TRI strategy and adjuvant ballooning were independent predictors for lower incidence of repeat revascularization during the 12-month of follow-up (Table 5). 
Table 1. Baseline clinical characteristics and laboratory findings

\begin{tabular}{|c|c|c|c|c|c|c|}
\hline \multirow[b]{2}{*}{ Characteristic } & \multicolumn{3}{|c|}{ Entire patients } & \multicolumn{3}{|c|}{ Matched patients } \\
\hline & $\begin{array}{c}\text { Radial } \\
(\mathrm{n}=220)\end{array}$ & $\begin{array}{l}\text { Femoral } \\
(\mathrm{n}=469)\end{array}$ & $p$ value & $\begin{array}{l}\text { Radial } \\
(\mathrm{n}=171)\end{array}$ & $\begin{array}{c}\text { Femoral } \\
(\mathrm{n}=171)\end{array}$ & $p$ value \\
\hline Male sex & $182(82.7)$ & $359(76.5)$ & 0.065 & $140(81.8)$ & $140(81.8)$ & NS \\
\hline Age, yr & $62.7 \pm 12.2$ & $61.8 \pm 12.4$ & 0.432 & $61.9 \pm 12.4$ & $62.9 \pm 12.0$ & 0.399 \\
\hline \multicolumn{7}{|l|}{ LV ejection fraction, \% } \\
\hline Before procedure & $50.3 \pm 9.5$ & $50.6 \pm 10.1$ & 0.804 & $50.0 \pm 9.5$ & $51.7 \pm 10.7$ & 0.250 \\
\hline After procedure & $51.2 \pm 10.3$ & $52.1 \pm 11.1$ & 0.300 & $51.1 \pm 9.9$ & $52.1 \pm 10.2$ & 0.344 \\
\hline \multicolumn{7}{|l|}{ Past history } \\
\hline Hypertension & $113(51.3)$ & $165(35.1)$ & $<0.001$ & $81(47 \cdot 3)$ & $83(48.5)$ & 0.829 \\
\hline Diabetes & $59(26.8)$ & $85(18.1)$ & 0.009 & $40(23 \cdot 3)$ & $37(21.6)$ & 0.698 \\
\hline Dyslipidemia & $57(25 \cdot 9)$ & $103(21.9)$ & 0.253 & $45(26.3)$ & $46(26.9)$ & 0.903 \\
\hline Stroke & $17(7.7)$ & $14(2.9)$ & 0.005 & $12(7 \cdot 0)$ & $11(6.4)$ & 0.829 \\
\hline Heart failure & $4(1.8)$ & $8(1.7)$ & NS & $3(1.7)$ & $4(2.3)$ & NS \\
\hline Peripheral artery disease & $3(1.3)$ & $1(0.2)$ & 0.098 & $1(0.5)$ & $1(0.5)$ & NS \\
\hline Chronic kidney disease & $4(1.8)$ & $8(1.7)$ & NS & $2(1.1)$ & $2(1.1)$ & NS \\
\hline History of smoking & $143(65.0)$ & $202(43.0)$ & $<0.001$ & $109(63.7)$ & $104(60.8)$ & 0.577 \\
\hline Current smokers & $115\left(5^{2.2}\right)$ & $169(36.0)$ & $<0.001$ & $87(50.8)$ & $84(49.1)$ & 0.746 \\
\hline Prior myocardial infarction & $9(4 \cdot 0)$ & $10(2.1)$ & 0.143 & $5(2.9)$ & $3(1.7)$ & 0.723 \\
\hline Prior PCI & $12(5.4)$ & $18(3.8)$ & 0.332 & $8(4.6)$ & $10(5.8)$ & 0.628 \\
\hline \multicolumn{7}{|l|}{ Laboratory findings } \\
\hline Hemoglobin, mg/dL & $14 \cdot 3 \pm 1.8$ & $14.1 \pm 1.8$ & 0.144 & $14 \cdot 3 \pm 1.8$ & $14.1 \pm 1.9$ & 0.377 \\
\hline CK-MB, mg/dL & $64 \pm 181$ & $43 \pm 95$ & 0.889 & & $27 \pm 59$ & 0.105 \\
\hline Troponin T, ng/dL & $1.0 \pm 2.6$ & $1.0 \pm 2.4$ & 0.247 & $1.2 \pm 3.0$ & $1.2 \pm 2.5$ & 0.454 \\
\hline Creatinine, mg/dL & $0.9 \pm 0.3$ & $1.0 \pm 0.5$ & 0.750 & $0.9 \pm 0.2$ & $0.9 \pm 0.3$ & 0.788 \\
\hline Total cholesterol, mg/dL & $179 \pm 44$ & $182 \pm 40$ & 0.263 & $178 \pm 44$ & $180 \pm 36$ & 0.735 \\
\hline Triglyceride, mg/dL & $121 \pm 85$ & $115 \pm 87$ & 0.108 & $119 \pm 84$ & $115 \pm 81$ & 0.510 \\
\hline HDL-C, mg/dL & $40 \pm 11$ & $40 \pm 9$ & 0.657 & $41 \pm 11$ & $39 \pm 8$ & 0.510 \\
\hline LDL-C, mg/dL & $113 \pm 36$ & $117 \pm 35$ & 0.256 & $113 \pm 38$ & $115 \pm 33$ & 0.693 \\
\hline Serum glucose, mg/dL & $160 \pm 64$ & $169 \pm 68$ & 0.100 & $159 \pm 65$ & $171 \pm 72$ & 0.046 \\
\hline Hemoglobin Aic, \% & $6.4 \pm 1.3$ & $6.5 \pm 1.5$ & 0.660 & $6.4 \pm 1.2$ & $6.6 \pm 1.7$ & 0.441 \\
\hline
\end{tabular}

Values are presented as number (\%) or mean $\pm \mathrm{SD}$.

NS, not significant (> 0.999); LV, left ventricle; PCI, percutaneous coronary intervention; CK-MB, creatine kinase-MB; HDL-C, high density lipoprotein cholesterol; LDL-C, low density lipoprotein cholesterol.

\section{DISCUSSION}

The main finding of the present study was that TRI for Korean STEMI patients undergoing a primary PCI with DES was associated with lower incidents of vascular access site complications, less in-hospital complications, less TVR, and less MACE in despite of longer procedure time and larger amount of contrast were needed during the 12-month of follow-up as compared to TFI.

The disadvantages of TRI include difficulty in learning the technique and a small radial artery size to work with. However, if the operator is experienced with routine TRI procedures in elective cases, TRI in acute myocardial infarction setting would be deemed safe and feasible. TRI can contribute to early ambulation and discharge [10]. Delarche et al. [11] have reported that TRI can 
Table 2. Baseline angiographic and procedural characteristics

\begin{tabular}{|c|c|c|c|c|c|c|}
\hline \multirow[b]{2}{*}{ Characteristic } & \multicolumn{3}{|c|}{ Entire patients } & \multicolumn{3}{|c|}{ Matched patients } \\
\hline & $\begin{array}{c}\text { Radial } \\
(\mathrm{n}=\mathbf{2 2 0})\end{array}$ & $\begin{array}{l}\text { Femoral } \\
(\mathrm{n}=469)\end{array}$ & $p$ value & $\begin{array}{c}\text { Radial } \\
(\mathrm{n}=171)\end{array}$ & $\begin{array}{c}\text { Femoral } \\
(\mathrm{n}=171)\end{array}$ & $p$ value \\
\hline Multi-vessel disease & $31(14.0)$ & $130(27.7)$ & $<0.001$ & $28(16.3)$ & $31(18.1)$ & 0.668 \\
\hline No. of treat vessels & $1.3 \pm 0.6$ & $1.4 \pm 0.6$ & 0.054 & $1.3 \pm 0.6$ & $1.2 \pm 0.4$ & 0.816 \\
\hline \multicolumn{7}{|l|}{ Target lesion } \\
\hline LAD & $134(60.9)$ & $290(61.8)$ & 0.816 & $106(61.9)$ & $113(66)$ & 0.430 \\
\hline LCX & $22(10.0)$ & $82(17 \cdot 4)$ & 0.011 & $21(12.2)$ & $11(6.4)$ & 0.063 \\
\hline RCA & $94(42.7)$ & $222(47 \cdot 3)$ & $0.25^{8}$ & $71(41.5)$ & $73(42.6)$ & 0.827 \\
\hline Left main & $2(0.9)$ & $12(2.5)$ & 0.245 & $2(1.1)$ & $3(1.7)$ & NS \\
\hline Ramus & $2(0.9)$ & $1(0.2)$ & 0.240 & $1(0.5)$ & $1(0.5)$ & NS \\
\hline Location & & & 0.841 & & & 0.144 \\
\hline Ostium & $125(56.8)$ & $273(58.2)$ & & $96(56.1)$ & $107(62.5)$ & \\
\hline Proximal & $61(27.7)$ & $130(27.7)$ & & $47(27 \cdot 4)$ & $49(28.6)$ & \\
\hline Mid & $28(12.7)$ & $5^{8}(12.3)$ & & $23(13.4)$ & $14(8.1)$ & \\
\hline Distal & $6(2.7)$ & $8(1.7)$ & & $5(2.9)$ & $1(0.5)$ & \\
\hline \multicolumn{7}{|l|}{ Lesion characteristics } \\
\hline Type (B2/C) & $165(75.0)$ & $435(92.7)$ & $<0.001$ & $142(83.0)$ & $144(84.2)$ & 0.770 \\
\hline Bifurcation & $44(20.0)$ & $118(25 \cdot 1)$ & 0.136 & $35(20.4)$ & $37(21.6)$ & 0.791 \\
\hline Diffuse (> $20 \mathrm{~mm})$ & $77(35.0)$ & $184(39.2)$ & 0.286 & $61(35.6)$ & $64(37 \cdot 4)$ & 0.736 \\
\hline Calcification & $25(11.3)$ & $32(6.8)$ & 0.044 & $21(12.2)$ & $21(12.2)$ & NS \\
\hline Chronic total occlusion & $30(13.6)$ & $37(7.8)$ & 0.018 & $23(13 \cdot 4)$ & $25(14.6)$ & 0.756 \\
\hline \multicolumn{7}{|l|}{ Stent type } \\
\hline Sirolimus-eluting & $10(4 \cdot 5)$ & $58(12.3)$ & 0.001 & $9(5.2)$ & $14(8.1)$ & 0.280 \\
\hline Paclitaxel-eluting & $85(38.6)$ & $188(40.0)$ & 0.717 & $66(38.5)$ & $68(39.7)$ & 0.825 \\
\hline Zotarolimus-eluting & $93(42.2)$ & $167(35 \cdot 6)$ & 0.092 & $77(45 \cdot 0)$ & $69(40.3)$ & 0.382 \\
\hline Everolimus-eluting & $82(37.2)$ & $177(37 \cdot 7)$ & 0.906 & $61(35.6)$ & $61(35.6)$ & NS \\
\hline \multicolumn{7}{|l|}{ Procedural characteristics } \\
\hline Sheath size, Fr & $5.9 \pm 0.3$ & $6.8 \pm 0.3$ & $<0.001$ & $5.9 \pm 0.3$ & $6.8 \pm 0.3$ & $<0.001$ \\
\hline Use of closing devices & 0 & $223(47 \cdot 5)$ & $<0.001$ & 0 & $57(33 \cdot 3)$ & $<0.001$ \\
\hline Image and FFR guided & $110(50.0)$ & $54(11.5)$ & $<0.001$ & $83(48.5)$ & $25(14.6)$ & $<0.001$ \\
\hline IVUS & $91(41.3)$ & $45(9.5)$ & $<0.001$ & $67(39.1)$ & $23(13 \cdot 4)$ & $<0.001$ \\
\hline FFR & $20(9.0)$ & $9(1.9)$ & $<0.001$ & $17(9.9)$ & $2(1.1)$ & $<0.001$ \\
\hline Adjuvant balloon inflation & $163(74.0)$ & $213(45 \cdot 4)$ & $<0.001$ & $131(76.6)$ & $70(40.9)$ & $<0.001$ \\
\hline Pre-loading of clopidogrel & $206(93.6)$ & $455(97.0)$ & 0.036 & $159(92.9)$ & $166(97.0)$ & 0.082 \\
\hline Loading dose, mg & $600(300-600)$ & $600(450-600)$ & 0.002 & $600(300-600)$ & $600(600-600)$ & 0.003 \\
\hline High-loading (>300 mg) & $136(61.8)$ & $279(59.4)$ & 0.560 & $103(60.2)$ & $123(71.9)$ & 0.022 \\
\hline Pre-loading of cilostazol & $13(5 \cdot 9)$ & $40(8.5)$ & 0.229 & $10(5.8)$ & $14(8.1)$ & 0.397 \\
\hline Low molecular weight heparin & $37(16.8)$ & $60(12.7)$ & 0.157 & $29(16.9)$ & $25(14.6)$ & 0.553 \\
\hline Glycoprotein IIb/IIIa inhibitors & $44(20.0)$ & $100(21.3)$ & 0.691 & $34(19.8)$ & $43(25.1)$ & 0.244 \\
\hline Contrast volume, cc & $225.6 \pm 82.8$ & $220.8 \pm 84 \cdot 3$ & 0.782 & $237 \cdot 9 \pm 87 \cdot 9$ & $216.1 \pm 95.0$ & 0.048 \\
\hline Procedure time, $\min$ & $41(31-55)$ & $35(25-50)$ & $<0.001$ & $43(31-55)$ & $40(25-50)$ & 0.003 \\
\hline Fluoroscopic time, min & $12(9-19)$ & $10(6-15)$ & 0.003 & $13(9-20)$ & $10(7-16)$ & 0.019 \\
\hline
\end{tabular}

Values are presented as number (\%), mean $\pm \mathrm{SD}$, or median (interquartile range).

LAD, left anterior descending; LCX, left circumflex; RCA, right coronary artery; NS, not significant (> 0.999); FFR, fractional flow reserve; IVUS, intravascular ultrasound. 
Table 3. Primary end-points: complications and cumulative 30-day clinical outcomes

\begin{tabular}{|c|c|c|c|c|c|c|}
\hline \multirow[b]{2}{*}{ Variable } & \multicolumn{3}{|c|}{ Entire patients } & \multicolumn{3}{|c|}{ Matched patients } \\
\hline & $\begin{array}{c}\text { Radial } \\
(\mathrm{n}=220)\end{array}$ & $\begin{array}{l}\text { Femoral } \\
(n=469)\end{array}$ & $p$ value & $\begin{array}{c}\text { Radial } \\
(\mathrm{n}=171)\end{array}$ & $\begin{array}{c}\text { Femoral } \\
(\mathrm{n}=171)\end{array}$ & $p$ value \\
\hline Procedural complications & $35(15.9)$ & $65(13.8)$ & 0.476 & $25(14.6)$ & $26(15.2)$ & 0.879 \\
\hline Dissection & $2(0.9)$ & $14(2.9)$ & 0.092 & $1(0.5)$ & $5(2.9)$ & 0.215 \\
\hline Perforation & $2(0.9)$ & $2(0.4)$ & 0.596 & $1(0.5)$ & $1(0.5)$ & NS \\
\hline Acute thrombosis & $2(0.9)$ & $3(0.6)$ & 0.657 & $2(1.1)$ & $2(1.1)$ & NS \\
\hline Distal embolization & $2(0.9)$ & $2(0.4)$ & 0.596 & $1(0.5)$ & $2(1.1)$ & NS \\
\hline No reflow & $16(7.2)$ & $27(5.7)$ & 0.443 & $11(6.4)$ & $10(5.8)$ & 0.822 \\
\hline Side branch occlusion & $11(5.0)$ & $15(3.1)$ & 0.247 & $9(5.2)$ & $5(2.9)$ & 0.275 \\
\hline \multicolumn{7}{|l|}{ Access site complications } \\
\hline Dissection & o & $1(0.2)$ & NS & 0 & $1(0.5)$ & NS \\
\hline Hematoma & 0 & $17(3.6)$ & 0.004 & o & $8(4.6)$ & 0.007 \\
\hline Major $(\geq 4 \mathrm{~cm})$ & o & $3(0.6)$ & 0.555 & o & $2(1.1)$ & 0.499 \\
\hline $\operatorname{Minor}(<4 \mathrm{~cm})$ & 0 & $14(2.9)$ & 0.007 & 0 & $6(3.5)$ & 0.030 \\
\hline \multicolumn{7}{|l|}{ In-hospital complications } \\
\hline Cardiogenic shock & $6(2.7)$ & $12(2.5)$ & 0.897 & $4(2.3)$ & $4(2.3)$ & NS \\
\hline Acute renal failure & $2(0.9)$ & $2(0.4)$ & 0.596 & $1(0.5)$ & $0(0.0)$ & NS \\
\hline Acute heart failure & $2(0.9)$ & $5(1.0)$ & NS & $1(0.5)$ & $2(1.1)$ & NS \\
\hline Stroke & $1(0.4)$ & $3(0.6)$ & NS & $1(0.5)$ & $1(0.5)$ & NS \\
\hline Gastrointestinal bleeding & $1(0.4)$ & $1(0.2)$ & 0.537 & $1(0.5)$ & $\mathrm{O}$ & NS \\
\hline Transfusion & $6(2.7)$ & $19(4.0)$ & 0.386 & $3(1.7)$ & $7(4 \cdot 0)$ & 0.199 \\
\hline Transfusion (Pint) & $3 \cdot 3 \pm 2.1$ & $4 \cdot 4 \pm 7 \cdot 5$ & 0.623 & $3 \cdot 3 \pm 1.1$ & $3.0 \pm 3.1$ & 0.197 \\
\hline Contrast reaction & $7(3.1)$ & $6(1.2)$ & 0.129 & $3(1.7)$ & $1(0.5)$ & 0.623 \\
\hline Contrast induced nephropathy & $6(2.7)$ & $4(0.8)$ & 0.082 & $2(1.1)$ & $1(0.5)$ & NS \\
\hline \multicolumn{7}{|l|}{ 30-Day outcomes after procedure } \\
\hline Total death & $6(2.7)$ & $10(2.1)$ & 0.629 & $4(2.3)$ & $2(1.1)$ & 0.685 \\
\hline Cardiac death & $4(1.8)$ & $9(1.9)$ & NS & $2(1.1)$ & $2(1.1)$ & NS \\
\hline Recurrent MI & ० & $3(0.6)$ & 0.555 & 0 & $1(0.5)$ & NS \\
\hline NSTEMI & o & $3(0.6)$ & 0.555 & o & $1(0.5)$ & NS \\
\hline Revascularizations & 0 & $6(1.2)$ & 0.184 & 0 & $2(1.1)$ & 0.499 \\
\hline Target lesion & o & $6(1.2)$ & 0.184 & 0 & $2(1.1)$ & 0.499 \\
\hline Target vessel & o & $6(1.2)$ & 0.184 & o & $2(1.1)$ & 0.499 \\
\hline Stent thrombosis & o & $5(1.0)$ & 0.183 & - & - & - \\
\hline
\end{tabular}

Values are presented as number (\%) or mean \pm SD.

NS, not significant (> 0.999); MI, myocardial infarction; NSTEMI, non-ST segment elevation MI.

be performed safely and effectively. Patients undergoing diagnostic cardiac catheterization are recommended to have transradial access because TRI can reduce hospital costs and improve the quality of life of patients after the procedure [12]. Several studies have reported that
TRI offers benefits to STEMI patients. However, no such benefit of TRI has been observed for patients with NSTEMI [13]. The primary concern is the learning curve associated with TRI access [14,15].

We have shown that even for an inexperienced TRI 
Table 4. Secondary end-points: cumulative 1-year major clinical outcomes

\begin{tabular}{|c|c|c|c|c|c|c|}
\hline \multirow[b]{2}{*}{ Variable } & \multicolumn{3}{|c|}{ Entire patients } & \multicolumn{3}{|c|}{ Matched patients } \\
\hline & $\begin{array}{c}\text { Radial } \\
(\mathrm{n}=\mathbf{2 2 0})\end{array}$ & $\begin{array}{l}\text { Femoral } \\
(\mathrm{n}=469)\end{array}$ & $p$ value & $\begin{array}{c}\text { Radial } \\
(\mathrm{n}=171)\end{array}$ & $\begin{array}{c}\text { Femoral } \\
(\mathrm{n}=171)\end{array}$ & $p$ value \\
\hline Total death & $11(5.0)$ & $17(3.6)$ & 0.394 & $7(4.0)$ & $6(3 \cdot 5)$ & 0.777 \\
\hline Cardiac death & $4(1.8)$ & $12(2.5)$ & 0.547 & $2(1.1)$ & $4(2.3)$ & 0.685 \\
\hline Recurrent MI & $\mathrm{o}$ & $12(2.5)$ & 0.012 & o & $4(2.3)$ & 0.123 \\
\hline STEMI & o & $2(0.4)$ & NS & 0 & $1(0.5)$ & NS \\
\hline NSTEMI & $\mathrm{O}$ & $10(2.1)$ & 0.035 & $\mathrm{O}$ & $3(1.7)$ & 0.248 \\
\hline Revascularizations & $2(0.9)$ & $25(5 \cdot 3)$ & 0.005 & $1(0.5)$ & $11(6.4)$ & 0.003 \\
\hline Target lesion & $2(0.9)$ & $17(3.6)$ & 0.042 & $1(0.5)$ & $6(3.5)$ & 0.121 \\
\hline Target vessel & $2(0.9)$ & $25(5 \cdot 3)$ & 0.005 & $1(0.5)$ & $11(6.4)$ & 0.003 \\
\hline Stent thrombosis & o & $6(1.2)$ & 0.184 & o & $1(0.5)$ & NS \\
\hline Stoke & 0 & $2(0.4)$ & NS & 0 & $2(1.1)$ & 0.499 \\
\hline MACE & $13(5 \cdot 9)$ & $45(9.5)$ & 0.104 & $8(4 \cdot 6)$ & $20(11.6)$ & 0.018 \\
\hline
\end{tabular}

Values are presented as number (\%). Univariate Cox proportional hazard analysis.

MI, myocardial infarction; STEMI, ST segment elevation MI; NS, not significant (> 0.999); NSTEMI, non-STEMI; MACE, major adverse cardiac event.

Table 5. Hazard ratio of transradial intervention for 12-month cumulative clinical outcomes compared with transfemoral intervention

\begin{tabular}{|c|c|c|c|c|}
\hline \multirow{2}{*}{ Variable } & \multicolumn{2}{|c|}{ Entire patients } & \multicolumn{2}{|c|}{ Matched patients } \\
\hline & Hazard ratio $(95 \%$ CI $)$ & $p$ value & Hazard ratio (95\% CI) & $p$ value \\
\hline Total death & $1.50(0.70-3.20)$ & 0.294 & $1.28(0.43-3.83)$ & 0.648 \\
\hline Cardiac death & $0.76(0.24-2.35)$ & 0.636 & $0.54(0.09-2.97)$ & 0.484 \\
\hline Recurrent MI & $0.02(0.00-5.46)$ & 0.185 & $0.01(0.00-52.4)$ & 0.319 \\
\hline Revascularizations & $0.18(0.04-0.79)$ & 0.023 & $0.09(0.01-0.75)$ & 0.026 \\
\hline Target lesion & $0.27(0.06-1.18)$ & 0.084 & $0.17(0.02-1.48)$ & 0.111 \\
\hline Target vessel & $0.18(0.04-0.79)$ & 0.023 & $0.09(0.01-0.76)$ & 0.026 \\
\hline MACE & $0.66(0.35-1.23)$ & 0.197 & $0.42(0.18-0.96)$ & 0.042 \\
\hline
\end{tabular}

Univariate Cox proportional hazard analysis.

$\mathrm{CI}$, confidence interval; MI, myocardial infarction; MACE, major adverse cardiac event.

operator, the door-to-balloon and catheterization laboratory door-to-balloon times were acceptable at the outset. They were and improved significantly over the course of the study.

The success rates for the two groups were high. The proportions of patients with TIMI grade 3 flow at the end of the procedure were comparable between the two groups. In our study, the choice of the access site was entirely dependent on the operator. The presence of hemodynamic instability, arrhythmias at the time of STEMI diagnosis, and cardiogenic shock were not con- traindicators for the transradial approach.

Since its introduction, TRI has gained more popularity due to its convenience and association with fewer complications [16]. The RIVAL trial and a meta-analysis haves shown that TRI is associated with a significant reduction of vascular complications [7]. Coronary stenting through the femoral route in acute coronary syndrome (ACS) is associated with more vascular complications. These complications are predictors of higher morbidity, longer hospital stays, and higher hospital costs $[17,18]$. In ST-Segment Elevation Myocardial Infarction Treated 
by Radial or Femoral Approach in a Multicenter Randomized Clinical (STEMI-RADIAL) trial, radial access is associated with a superior net clinical benefit and less vascular complications than femoral access [19]. With a trend toward a shorter length of hospitalization and fewer vascular complications, TRI appears to be more effective than TFI. The door-to-balloon time is shorter with the femoral approach. The choice of sheath is less restrictive with this approach. In addition, vascular closure devices can yield better clinical outcomes [7,14]. A meta-analysis has shown that closure devices may reduce bleeding complications and mortality; thereby, filling the gap between the two access sites [15].

It should be kept in mind that current literatures supporting radial access over femoral access have been reduced from studies involving suboptimal use of antithrombotic agents and unrestricted use of parenteral antiplatelet agents. Hence, a randomized study involving patients treated with bivalirudin or newer antiplatelets, and an appropriate use of anticoagulation regimens without adjunctive GP inhibitors is needed to determine access site advantage in STEMI patient.

In our study, even though the two groups showed no difference regarding in-hospital complications or mortality, local vascular complications and hematoma occurred solely in the TFI group, irrespective of whether a closure device was used. The incidence rates of access site complications and bleeding complications were significantly lower in the TRI group. In our study, higher loading dose of clopidogrel and use of a larger sheath were observed in the TFI group. Access site complications showed higher incidence of minor hematoma in the TFI group, which was belong to anatomical specificities in the TFI group treated with larger sheath, but this was not a serious complication affecting the major clinical outcomes including mortality rate. The device size and diameter used in the TFI procedure were significantly reduced and the performance of closure devices was more improved compared to the past. Thus, we think anatomic specificities between TFI and TRI did not reach in differences in major bleeding. However, exposure to radiation was higher in the TRI group than that in the TFI group in the RIVAL study, although the difference was only observed in low volume centers and operators [20] consistent with our study results showing that contrast amount in the TRI group was higher while procedure time and fluoroscopic time were longer in the TRI group than those in the TFI group.

Previous study have shown that the crossover rate from TRI to TFI access is approximately $9.8 \%$ [14]. Our crossover rate was $10 \%$. This might reflect the initial limited experience of TRI operators as there was no more crossover during the last 12 months of the study.

The RIVAL trial results (published while our study was still in progress) showed that the primary outcomes of death, MI, stroke, and non-coronary artery bypass grafting bleeding were not significantly different between the two groups in the ACS population. However, in the subset of STEMI population, mortality rate was lower in the radial group compared to that in the femoral group. In Radial versus Femoral Randomized Investigation in ST-segment Elevation Acute Coronary Syndrome (RIFLE-STEACS) study, the radial approach reduced the rate of primary outcomes of cardiac death, stroke, MI, TLR, and bleeding during a 30-day follow-up compared to the femoral approach (13.6\% vs. $21.0 \%, p=$ 0.003 ) and MACE [14]. In our study, despite the two groups had similar incidence of mortality, the incidence of TVR and MACE were lower in the TRI group compared to those in the TFI group during the 12-month of follow-up. Although the baseline differences including multivessel diseases and CTO lesions were adjusted by PSM, still the TVR rate was lower in the TRI group than in the TFI group. The complex lesion subset and complex patients in the TFI group might not be perfectly adjusted by statistics because this result is not a randomized data. Furthermore, image-guided PCI was more frequently performed in the TRI group. We speculated that a higher incidence of image-guided PCI in the TRI group might lead to a higher incidence of subsequent adjuvant ballooning based on imaging findings to achieve stent optimization; thus, reducing the number of cases/episodes of stent malapposition and subsequent repeat revascularization. We think that the choice of TRI and TFI route was based on the individual operator's discretion and it did not have a direct impact on the outcome difference in our study.

In this study, TLR was included in the definition of TVR, and TLR also showed numerically lower in the TRI group although the difference was not statistically significant. In entire patients before PSM, the incidence of repeat revascularization including TLR and TVR was 
higher in the TFI group when compared with the TRI group during the 12-month follow-up. However, after the PSM analysis, the incidence of recurrent MI and TLR was similar between the two groups.

Instead of non-TVR, TVR difference is also important as TLR because the definition of TVR includes both TLR and non-TLR TVR. The TVR incidence includes in-stent, in-segment for both stent edges and non-target lesion progression. Thus, TVR is also an important component of repeat revascularization and should be interpreted as a major component of MACE. Our clinical endpoints are primarily focused on patient-based clinical outcomes rather than lesion-based clinical outcomes such as target-lesion failure.

The incidence of MACE was significantly lower in the TRI group than that in the TFI group mainly due to significantly lower incidence of TVR in the TRI group compared to that in the TFI group.

The present study has certain limitations. It was a retrospective observational study. Therefore, results must be interpreted with caution as technical differences among operators in different PCI centers might have caused biases. In addition, the in-hospital outcome results might have limitation since factors such as bleeding score was not recorded in the data due to the fact that this was a multicenter study without scheduled prospective protocol. Although the number of participants was relatively small to reach a definite final conclusion, radial access was found to have definite advantage in this study as in previous studies. This is clinically important implications for Korean population. Even though PSM was performed to eliminate biases, some unmeasurable confounders might have influenced the outcomes of the study.

In conclusion, TRI in STEMI patients undergoing primary PCI with DESs was associated with lower incidence of vascular-access site hematomas, lower 12-month TVR, and lower incidence of MACE despite of longer procedure time and larger amount of contrast needed in our study. There was no significant difference in in-hospital or 12-month mortality rate between the two groups. Our results suggest that TRI might play an important role in reducing access site vascular complications and improving mid-term major clinical outcomes in STEMI patients undergoing primary PCI with DES in Korean population.

\section{KEY MESSAGE}

1. Transradial intervention (TRI) in ST-elevation myocardial infarction patients undergoing primary percutaneous coronary intervention was associated with lower incidence of access site hematoma, 12-month repeat revascularization, and major adverse cardiac event compared to transfemoral intervention.

2.TRI in acute myocardial infarction setting would be deemed safe and feasible.

3. TRI can contribute to early ambulation and discharge.

\section{Conflict of interest}

No potential conflict of interest relevant to this article was reported.

\section{REFERENCES}

1. Agostoni P, Biondi-Zoccai GG, de Benedictis ML, et al. Radial versus femoral approach for percutaneous coronary diagnostic and interventional procedures: systematic overview and meta-analysis of randomized trials. J Am Coll Cardiol 2004;44:349-356.

2. Jolly SS, Amlani S, Hamon M, Yusuf S, Mehta SR. Radial versus femoral access for coronary angiography or intervention and the impact on major bleeding and ischemic events: a systematic review and meta-analysis of randomized trials. Am Heart J 2009;157:132-140.

3. Kinnaird TD, Stabile E, Mintz GS, et al. Incidence, predictors, and prognostic implications of bleeding and blood transfusion following percutaneous coronary interventions. Am J Cardiol 2003;92:930-935.

4. Yatskar L, Selzer F, Feit F, et al. Access site hematoma requiring blood transfusion predicts mortality in patients undergoing percutaneous coronary intervention: data from the National Heart, Lung, and Blood Institute Dynamic Registry. Catheter Cardiovasc Interv 2007;69:961966.

5. Oweida SW, Roubin GS, Smith RB 3rd, Salam AA. Postcatheterization vascular complications associated with percutaneous transluminal coronary angioplasty. J Vasc Surg 1990;12:310-315. 
6. Nasser TK, Mohler ER 3rd, Wilensky RL, Hathaway DR. Peripheral vascular complications following coronary interventional procedures. Clin Cardiol 1995;18:609-614.

7. Jolly SS, Yusuf S, Cairns J, et al. Radial versus femoral access for coronary angiography and intervention in patients with acute coronary syndromes (RIVAL): a randomised, parallel group, multicentre trial. Lancet 2011;307:1409-1420.

8. Mehran R, Rao SV, Bhatt DL, et al. Standardized bleeding definitions for cardiovascular clinical trials: a consensus report from the Bleeding Academic Research Consortium. Circulation 2011;123:2736-2747.

9. Cutlip DE, Windecker S, Mehran R, et al. Clinical end points in coronary stent trials: a case for standardized definitions. Circulation 2007;115:2344-2351.

10. Kiemeneij F, Laarman GJ, Odekerken D, Slagboom T, van der Wieken R. A randomized comparison of percutaneous transluminal coronary angioplasty by the radial, brachial and femoral approaches: the access study. J Am Coll Cardiol 1997;29:1269-1275.

11. Delarche N, Idir M, Estrade G, Leblay M. Direct angioplasty for acute myocardial infarction in elderly patients using transradial approach. Am J Geriatr Cardiol 1999;8:32-35.

12. Cooper CJ, El-Shiekh RA, Cohen DJ, et al. Effect of transradial access on quality of life and cost of cardiac catheterization: a randomized comparison. Am Heart J 1999;138:430-436.

13. Mehta SR, Jolly SS, Cairns J, et al. Effects of radial versus femoral artery access in patients with acute coronary syndromes with or without ST-segment elevation. J Am Coll Cardiol 2012;60:2490-2499.

14. Romagnoli E, Biondi-Zoccai G, Sciahbasi A, et al. Radial versus femoral randomized investigation in ST-segment elevation acute coronary syndrome: the RIFLE-STEACS (Radial Versus Femoral Randomized Investigation in ST-Elevation Acute Coronary Syndrome) study. J Am Coll Cardiol 2012;60:2481-2489.

15. Byrne RA, Cassese S, Linhardt M, Kastrati A. Vascular access and closure in coronary angiography and percutaneous intervention. Nat Rev Cardiol 2013;10:27-40.

16. Saito S, Tanaka S, Hiroe Y, et al. Comparative study on transradial approach vs. transfemoral approach in primary stent implantation for patients with acute myocardial infarction: results of the test for myocardial infarction by prospective unicenter randomization for access sites (TEMPURA) trial. Catheter Cardiovasc Interv 2003;59:2633 .

17. Ellis SG, Miller DP, Brown KJ, et al. In-hospital cost of percutaneous coronary revascularization: critical determinants and implications. Circulation 1995;92:741-747.

18. Wolfe MW, Roubin GS, Schweiger M, et al. Length of hospital stay and complications after percutaneous transluminal coronary angioplasty: clinical and procedural predictors. Heparin Registry Investigators. Circulation 1995;92:311-319.

19. Bernat I, Horak D, Stasek J, et al. ST-segment elevation myocardial infarction treated by radial or femoral approach in a multicenter randomized clinical trial: the STEMI-RADIAL trial. J Am Coll Cardiol 2014;63:964-972.

20. Jolly SS, Cairns J, Niemela K, et al. Effect of radial versus femoral access on radiation dose and the importance of procedural volume: a substudy of the multicenter randomized RIVAL trial. JACC Cardiovasc Interv 2013;6:258266. 